\title{
Status of the Dark Energy Survey Camera (DECam) Project
}

Brenna L. Flaugher ${ }^{\mathrm{a}}$, Timothy M. C. Abbott ${ }^{\mathrm{b}}$, Jim Annis ${ }^{\mathrm{a}}$, Michelle L. Antonik ${ }^{\mathrm{h}}$, Jim Bailey $^{\mathrm{c}}$, Otger Ballester ${ }^{\mathrm{k}}$, Joseph P. Bernstein ${ }^{\mathrm{c}}$, Rebbeca Bernstein ${ }^{\mathrm{m}}$, Marco Bonati ${ }^{\mathrm{b}}$, Gale Bremer ${ }^{\mathrm{b}}$, Jorge Briones $^{\mathrm{b}}$, David Brooks ${ }^{\mathrm{h}}$, Elizabeth J. Buckley-Geer ${ }^{\mathrm{a}}$, Juila Campa ${ }^{\mathrm{l}}$, Laia Cardiel-Sas ${ }^{\mathrm{k}}$, Franciso Castander $^{\mathrm{k}}$, Javier Castilla ${ }^{\mathrm{l}}$, Herman Cease ${ }^{\mathrm{a}}$, Steve Chappa ${ }^{\mathrm{a}}$, Edward C. Chi ${ }^{\mathrm{a}}$, Luis da Costa ${ }^{\mathrm{p}}$, Darren L. DePoy ${ }^{\mathrm{d}}$, Gregory Derylo ${ }^{\mathrm{a}}$, Juan de Vicente ${ }^{1}$, H. Thomas Diehl $^{\mathrm{a}}$, Peter Doel ${ }^{\mathrm{h}}$, Juan Estrada ${ }^{\mathrm{a}}$, Jacob Eiting $^{\mathrm{g}}$, Anne Elliott ${ }^{\mathrm{g}}$, David Finley ${ }^{\mathrm{a}}$, Josh Frieman ${ }^{\mathrm{a}}$, Enrique Gaztanaga ${ }^{\mathrm{k}}$, David Gerdes ${ }^{\mathrm{e}}$, Mike Gladders $^{n}$, V. Guarino ${ }^{c}$, G. Gutierrez ${ }^{\mathrm{a}}$, Jim Grudzinski ${ }^{\mathrm{c}}$, Bill Hanlon ${ }^{\mathrm{f}}$, Jiangang Hao ${ }^{\mathrm{a}}$, Steve Holland ${ }^{\mathrm{i}}$, Klaus Honscheid $^{\mathrm{g}}$, Dave Huffman ${ }^{\mathrm{a}}$, Cheryl Jackson ${ }^{\mathrm{a}}$, Inga Karliner ${ }^{\mathrm{f}}$, Daekwang Kau ${ }^{\mathrm{f}}$, Steve Kent ${ }^{\mathrm{a}}$, Kurt Krempetz ${ }^{\mathrm{a}}$, John Krider ${ }^{\mathrm{a}}$, Mark Kozlovsky ${ }^{\mathrm{a}}$, Donna Kubik ${ }^{\mathrm{a}}$, Kyler W. Kuehn ${ }^{\mathrm{c}}$, Stephen E. Kuhlmann $^{\mathrm{c}}$, Kevin Kuk ${ }^{\mathrm{a}}$, Ofer Lahav ${ }^{\mathrm{h}}$, Peter Lewis ${ }^{\mathrm{h}}$, Huan Lin ${ }^{\mathrm{a}}$, Wolfgang Lorenzon ${ }^{\mathrm{e}}$, Stuart Marshall ${ }^{\mathrm{j}}$, Gustavo Martínez ${ }^{1}$, Timothy McKay ${ }^{\mathrm{e}}$, Wyatt Merritt ${ }^{\mathrm{a}}$, Mark Meyer ${ }^{\mathrm{f}}$, Ramon Miquel ${ }^{1}$, Jim Morgan $^{\mathrm{c}}$, Peter Moore ${ }^{\mathrm{b}}$, Todd Moore ${ }^{\mathrm{f}}$, Brian Nord ${ }^{\mathrm{e}}$, R. Ogando ${ }^{\mathrm{p}}$, Jamieson Olsen ${ }^{\mathrm{a}}$, John Peoples $^{\mathrm{a}}$,

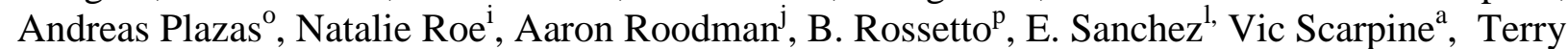
Schalk $^{\mathrm{m}}$, Rafe Schindler ${ }^{\mathrm{j}}$, Ricardo Schmidt ${ }^{\mathrm{b}}$, Richard Schmitt ${ }^{\mathrm{a}}$, Mike Schubnell ${ }^{\mathrm{e}}$, Kenneth Schultz ${ }^{\mathrm{a}}$, M. Selen ${ }^{\mathrm{f}}$, S. Serrano ${ }^{\mathrm{k}}$, Terri Shaw ${ }^{\mathrm{a}}$, Vaidis Simaitis ${ }^{\mathrm{f}}$, Jean Slaughter ${ }^{\mathrm{a}}$, R. Christopher Smith ${ }^{\mathrm{b}}$, Hal Spinka $^{c}$, Andy Stefanik ${ }^{\mathrm{a}}$, Walter Stuermer ${ }^{\mathrm{a}}$, Adam Sypniewski ${ }^{\mathrm{e}}$, Rick Talaga, Greg Tarle ${ }^{\mathrm{e}}$, Jon Thaler $^{\mathrm{f}}$, Doug Tucker ${ }^{\mathrm{a}}$, Alistair R. Walker ${ }^{\mathrm{b}}$, Curtis Weaverdyck ${ }^{\mathrm{e}}$, William Wester ${ }^{\mathrm{a}}$, Robert J. Woods $^{\mathrm{a}}$, Sue Worswick ${ }^{\mathrm{h}}$, Allen Zhao ${ }^{\mathrm{c}}$

${ }^{\mathrm{a}}$ Fermi National Accelerator Laboratory, PO Box 500, Batavia, IL, USA 60510;

${ }^{\mathrm{b}}$ Casilla 603, AURA/Cerro Tololo Inter-American Observatory, La Serena, Chile; ${ }^{c}$ Physics Dept., Argonne National Laboratory, 9700 S. Cass Ave., Argonne, IL 60439;

${ }^{\mathrm{d}}$ Dept. of Physics, Texas A\&M University, 4242 TAMU, College Station, TX USA 77843-4242;

${ }^{e}$ Physics Dept., University of Michigan, 450 Church St., Ann Arbor, MI, 48109-1040;

${ }^{\mathrm{f}}$ Dept. of Physics, University of Illinois, 1110 W. Green St., Urbana, IL 61801-3080;

${ }^{g}$ Dept. of Physics, The Ohio State University, 191 West Woodruff Ave., Columbus, OH 43210;

${ }^{\mathrm{h}}$ University College London, Gower Street, Bloomsbury, London WC1E 6BT, United Kingdom;

${ }^{\mathrm{i}}$ Lawrence Berkley National Lab, 1 Cyclotron Road, Berkeley, CA 94720;

${ }^{\mathrm{j} S t a n f o r d ~ L i n e a r ~ A c c e l e r a t o r ~ C e n t e r, ~} 2575$ Sand Hill Road, Menlo Park, CA 94025;

${ }^{\mathrm{k}}$ Institut de Física d'Altes Energies (IFAE) .Edifici Cn, Universitat Autònoma de Barcelona (UAB), E-08193 Bellaterra (Barcelona), Spain;

${ }^{1}$ Centro de Investigaciones Energéticas, Medioambientales y Tecnológias (CIEMAT), Avda. Complutense, 22 - 28040 (Madrid), Spain;

${ }^{\mathrm{m}} \mathrm{UC}$ Observatories \& Astrophysics Dept, UC Santa Cruz, CA;

${ }^{\mathrm{n}}$ University of Chicago, 5801 South Ellis Avenue, Chicago, IL 60637;

${ }^{\circ}$ University of Pennsylvania, 3451 Walnut Street, Philadelphia, PA 19104;

${ }^{\mathrm{p}}$ Observatório Nacional, Rua General José Cristino, 77 Bairro Imperial de São Cristóvão

Rio de Janeiro, RJ - Brasil CEP: 20921-400

For the Dark Energy Survey Collaboration

†Brenna@fnal.gov Fermi National Accelerator Laboratory, Phone: 1-630-840-2934 


\begin{abstract}
The Dark Energy Survey Collaboration is building the Dark Energy Camera (DECam), a 3 square degree, 520 Megapixel CCD camera which will be mounted on the Blanco 4-meter telescope at CTIO. DECam will be used to perform the $5000 \mathrm{sq}$. deg. Dark Energy Survey with $30 \%$ of the telescope time over a 5 year period. During the remainder of the time, and after the survey, DECam will be available as a community instrument. Construction of DECam is well underway. Integration and testing of the major system components has already begun at Fermilab and the collaborating institutions.
\end{abstract}

Keywords: Dark Energy, CCD, camera, survey, Blanco, CTIO

\title{
1. INTRODUCTION
}

The DES Collaboration formed in 2004 around a common interest pursuing the nature of dark energy and the NOAO announcement of opportunity that offered a significant amount of telescope time on the CTIO Blanco 4m telescope in exchange for a new state-of-the-art prime focus instrument (see www.darkenergysurvey.org). The Collaboration submitted a proposal to NOAO to build DECam, a wide field optical imager with thick fully depleted CCDs and a filter set optimized for obtaining precise photometric redshifts, and a science program aimed at making a significant improvement in the constraints on the dark energy equation of state. NOAO approved the proposal near the end of 2004 and the collaboration has been growing and developing the DECam instrument since that time. The DES collaboration now consists of approximately 120 scientists located at 4 US national laboratories, 7 US universities, NOAO/CTIO, and consortia from the United Kingdom, Spain, Brazil and Germany.

In Nov. 2005 DECam, the part of DES funded largely by the US department of Energy (DOE), received CD-0 approval (mission need) from DOE and in October 2008 received approval to begin full construction (CD-3). We anticipate that first light and commissioning of DECam on the Blanco will begin in October 2011. The cost of the DECam instrument is $\$ 35 \mathrm{M}$, provided by DOE, plus approximately $\$ 7 \mathrm{M}$ from the collaborating institutions. As outlined in the Announcement of Opportunity, NOAO has allocated the DES Collaboration 105 scheduled nights/year on the Blanco for 5 years to perform a deep multi-color survey covering 5000 degree $^{2}$ of the southern sky. DECam will be available as a community instrument when not in use by the DES Collaboration.

The area and location of the DES are chosen to overlap with the South Pole Telescope Project (SPT) (see pole.uchicago.edu/), which will provide Sunyaev-Zeldovich effect measurements for a large number of galaxy clusters. The five optical filter pass bands, g, r, i, z, and Y have been chosen to provide photometric redshifts for all the DES targets. The $\mathrm{Y}$ band filter has been included in the DES filter set under an agreement with the Vista project (see www.ast.cam.ac.uk/ rgm/vhs/ and www.eso.org/sci/observing/policies/PublicSurveys/sciencePublicSurveys.html). The DES will provide the Vista Hemispherical Survey (VHS) with Y band data, in exchange for near-infrared measurements in $\mathrm{J}, \mathrm{H}$ and $\mathrm{K}$ that improve photometric redshift precision and accuracy for the DES area.

The primary scientific goal of the DES is to measure the dark energy equation of state parameter $w$ using four independent and complementary techniques: galaxy cluster counting, baryon oscillations, weak lensing, and Type Ia supernovae. The large survey volume ( 300 million galaxies) will enable precise and complementary constraints on $\mathrm{w}$ from weak lensing and from measurements of baryon oscillations. By using $8 \%$ of the photometric time and $30 \%$ of the total DES survey time we expect to find $3000 \mathrm{SN}$ Ia out to a redshift of 1.2 which can provide another complementary constraint on w. The overlap with the SPT provides an independent and complementary measurement of the galaxy cluster masses which reduces the systematic uncertainties on this quantity. The DES constraints and systematic uncertainties are described in the DES whitepapers for the Dark Energy Task Force (see astro-ph/0510346, astro$\mathrm{ph} / 0510194$, and astro-ph/0510195; these are also available at https://www.darkenergysurvey.org/theproject/survey_documents/DES-DETF/ and supplements that are part of the Dark Energy Task Force (DETF) report). In the language of the DETF findings the DES falls into the category of a stage III project: near term and modest cost compared to the stage IV projects such as LSST and JDEM. We expect to improve the DETF figure-of-merit by a factor of 4.6 over current (stage II) projects and results. 


\section{DECAM}

The design of DECam was guided by the goal of maximizing the scientific potential while minimizing the construction time and cost. The DECam project also has a strong emphasis on integration and testing prior to installation in order to minimize the downtime of the Blanco telescope. Early in the project we constructed a full size prototype imager which has been extremely valuable for development and integration of the imager systems. We are currently constructing a replica of the top end of the Blanco at Fermilab which will allow assembly, integration and testing of all DECam components, except the optics, as a system and in all orientations. This telescope simulator will also allow testing of the installation fixtures and procedures for DECam with the same platforms and accessibility constraints as we will have at CTIO. See Testing the Dark Energy Camera on a Telescope Simulator submitted by Tom Diehl (Paper 7735-125) in these proceedings for more detail. Table 1 contains a summary of the DES survey parameters and the expected performance of the CTIO site and DECam.

The DES survey strategy is based on taking multiple short exposures $(100 \mathrm{sec})$ and adding them together to reach the required depth in each filter pass-band. This strategy minimizes the systematic uncertainties resulting from effects such as atmospheric variations. In first two years we plan to tile the entire survey area multiple times to enable early scientific results. To maximize the total time available for exposures, we plan to read out the image CCDs while the telescope is slewing to a new position. Current measurements indicate that the time it takes for a typical DES slew of 2 deg. is $\sim 35 \mathrm{sec}$. A CCD readout rate of $250 \mathrm{kpix} / \mathrm{sec}$ will take $17 \mathrm{sec}$ and fit within the telescope slew time. Upgrades to the Blanco should reduce the slew time to $\sim 20 \mathrm{sec}$. See Improving the Blanco Telescope's Delivered Image Quality submitted by T. Abbott (paper 7733-151) for more details.

Figure 2 shows a model of DECam and a picture of the Blanco telescope. DECam will replace the entire prime focus cage of the Blanco and attach to the existing spider support fins. The major components of DECam are a $\sim 520$ megapixel optical CCD camera with vacuum and cryogenic controls, a compact low noise CCD readout system housed in actively cooled crates, a shutter, a filter system to house and exchange the DES filters (g,r,i,z,Y) plus slots for three additional filters that could be provided by NOAO for the observer community, and a wide-field optical corrector (2.2 deg. field of view). The CCD vessel and corrector are supported as a single unit by a hexapod that will provide adjustability in all degrees of freedom including focus, lateral translations, tip, and tilt. The current prime focus cage of the Blanco can flip such that the F/8 mirror that is mounted to the back of the cage, behind the CCD vessel, points towards the primary mirror for Cassegrain observations. The DECam project will maintain the use of the F/8 by providing a handling system that can attach the secondary mirror to the front of the new DES prime focus cage.

Table 1: Expected performance of DECAM, Blanco, and CTIO site

\begin{tabular}{|c|c|}
\hline Blanco Effective Aperture/ f number @ prime focus & $4 \mathrm{~m} / 2.7$ \\
\hline Blanco Primary Mirror - $80 \%$ encircled energy & $0.25 \operatorname{arcsec}$ \\
\hline Optical Corrector Field of View & 2.2 deg. \\
\hline Corrector Wavelength Sensitivity & $<350-1050 \mathrm{~nm}$ \\
\hline Filters & $\mathrm{g}, \mathrm{r}, \mathrm{i}, \mathrm{z}, \mathrm{Y}(400-1050 \mathrm{~nm})$ \\
\hline Effective Area of CCD Focal Plane & 3.0 sq. deg. \\
\hline Image CCD pixel format/ total \# pixels & $2 \mathrm{~K}$ X 4K/ 520 Mpix \\
\hline Guide, Focus \& Alignment Sensor CCD pixel format & $2 \mathrm{~K} X 2 \mathrm{~K}$ \\
\hline Pixel Size & $0.27 \mathrm{arcsec} / 15 \mu \mathrm{m}$ \\
\hline Readout Speed/Noise requirement & $250 \mathrm{kpix} / \mathrm{sec} / 10 \mathrm{e}$ \\
\hline $\begin{array}{l}\text { Survey Area } \\
\text { SPT overlap } \\
\\
\text { SDSS stripe } 82 \\
\text { Connection region } \\
\end{array}$ & $\begin{array}{l}5,000 \text { sq. deg. total } \\
\text { RA }-60 \text { to } 105, \text { DEC }-30 \text { to }-65 \\
\text { RA }-30 \text { to } 30, \text { DEC }-30 \text { to }-25 \\
\text { RA }-50 \text { to } 55, \text { Dec }-1 \text { to } 1 \\
\text { RA } 30 \text { to } 55, \text { Dec }-30 \text { to }-1\end{array}$ \\
\hline Survey Time/Duration & $525 / 5$ (nights/years) \\
\hline Median Site Seeing Sept. - Feb. & $0.65 \operatorname{arcsec}$ \\
\hline Median Delivered Seeing with Mosaic II on the Blanco & $0.9-1.0$ arcsec $(\mathrm{V}$ band $)$ \\
\hline Limiting Magnitude: $10 \sigma$ in 1.6 " aperture assuming 0.9 " seeing, AB system & $\mathrm{g}=24.7, \mathrm{r}=24.2, \mathrm{i}=24.4, \mathrm{z}=23.9$ \\
\hline Limiting Magnitude: $5 \sigma$ for point sources assuming 0.9 " seeing, $\mathrm{AB}$ system & $\mathrm{g}=26.1, \mathrm{r}=25.6, \mathrm{i}=25.8, \mathrm{z}=25.3$ \\
\hline
\end{tabular}




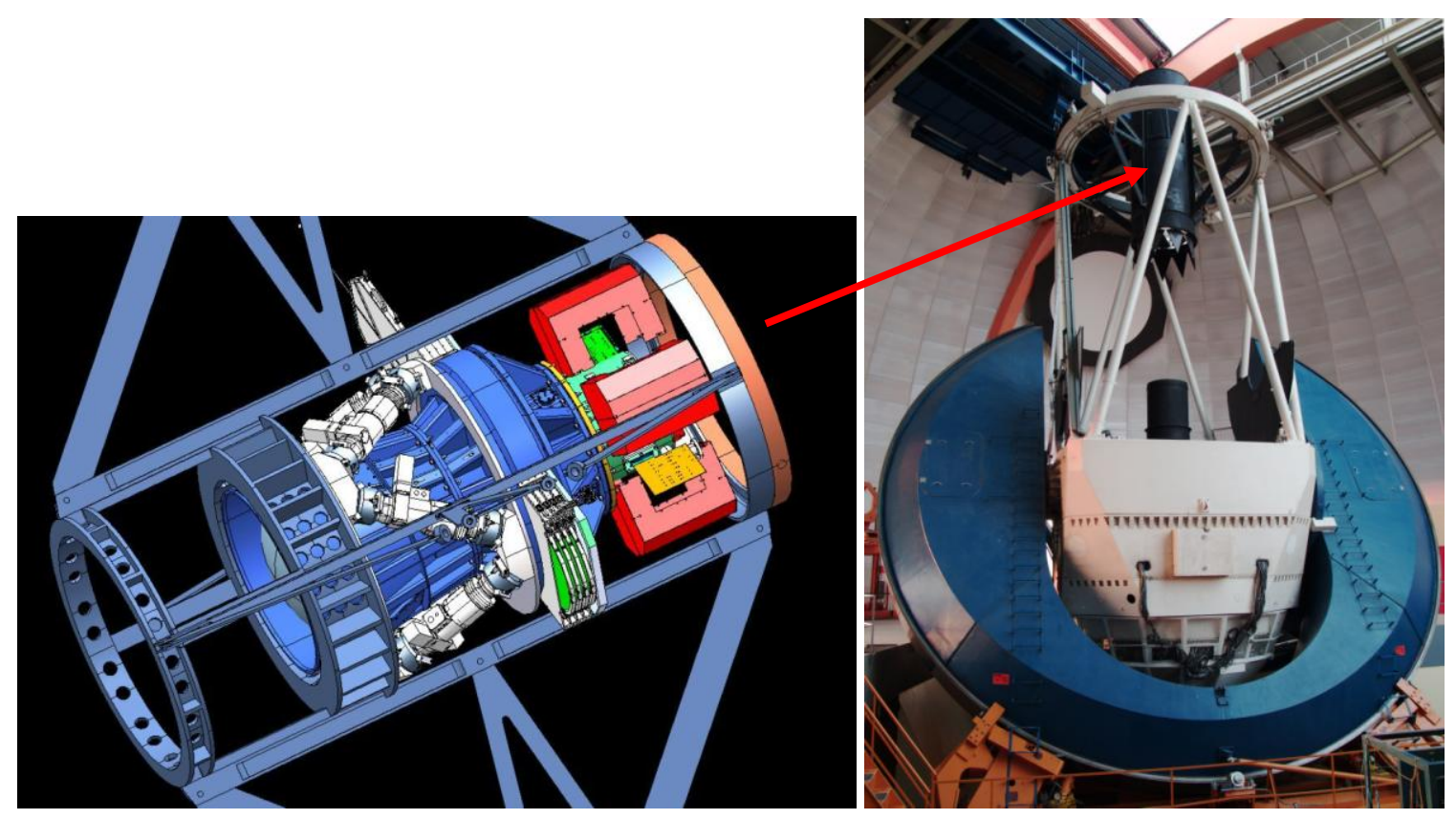

Figure 1: Schematic view of the DECam and a picture of the Blanco telescope. The arrow connecting the two shows the position of the Blanco telescope prime focus, where DECam will be mounted.

The DECam project first concentrated on the CCD fabrication and assembly and on the design and fabrication of the optical elements as these were identified as the highest risk (technical, cost and schedule) and longest lead-time aspects of the project. We currently have enough science quality CCDs on hand at Fermilab to fill the DECam focal plane. The CCD fabrication is discussed briefly below and in papers submitted to these proceedings. The DECam optical corrector has 5 fused silica lenses with 8 spheric and 2 aspheric surfaces. Corning provided the blanks in 2008 and in June of that year SESO began polishing the lenses. As of May 2010 polishing of two of the lenses is complete and coating has just begun. We anticipate completion of all the lenses in July 2010.

The design of all the major DECam systems is complete and fabrication is in progress or complete. Below we give an overview of these systems. Detailed papers have also been submitted to these proceedings on many aspects of DECam:

Focal plane detectors for the dark energy survey, submitted by Juan Estrada (Paper 7735-61)

Automated Characterization of CCD detectors for DECam, submitted by Donna Kubik (Paper 7735-311)

Assembly of the dark energy survey CCD imager submitted by Greg Derylo (Paper Number: 7739-163),

Measuring the flatness of focal plane for very large mosaic CCD camera submitted by Jiangang Hao (Paper Number: 7735-137)

Focus and Alignment using Out-of-focus Stellar Images at the Dark Energy Survey submitted by Aaron Roodman (Paper 7735-136),

System architecture of the dark energy survey camera readout electronics submitted by Terri Shaw (Paper 7735-123)

Readout Electronics for DECam submitted by Javier Castilla (Paper 7735-311) in these proceedings.

Cooling the dark energy camera CCD array using a closed-loop two-phase liquid nitrogen system submitted by Herman Cease(Paper Number: 7739-164), 
Large format filter changer mechanism and shutter for the dark energy survey submitted by Greg Tarle (Paper 7739-162),

Spectrophotometric calibration system for DECam submitted by JP Rheault (Paper 7735-226),

Design and initial performance of a radiometric all-sky infrared camera (RASICAM) for DES/CTIO submitted by Peter Lewis (Paper 7735-119),

The DECam data acquisition and control system submitted by Klaus Honscheid (Paper 7740-57) and

HTML 5, Websockets, and Sproutcore: a web-based user interface for the dark energy camera (DECam) submitted by Jacob Eiting (Paper 7740-39)

\subsection{DES Focal plane}

To efficiently obtain $z$-band images for high-redshift ( $\mathrm{z}$ 1.3) galaxies, we use fully depleted, high-resistivity, 250 micron thick CCDs [1] that have been designed and developed at the LBNL. The CCD thickness has two important implications for DES: fringing is negligible and the QE of these devices is > 50\% in the $z$ band, a factor of 5-10 higher than traditional thinned astronomical devices. Figure 2 shows the QE of the DES CCDs compared to the CCDs currently in the Mosaic II camera on the Blanco.

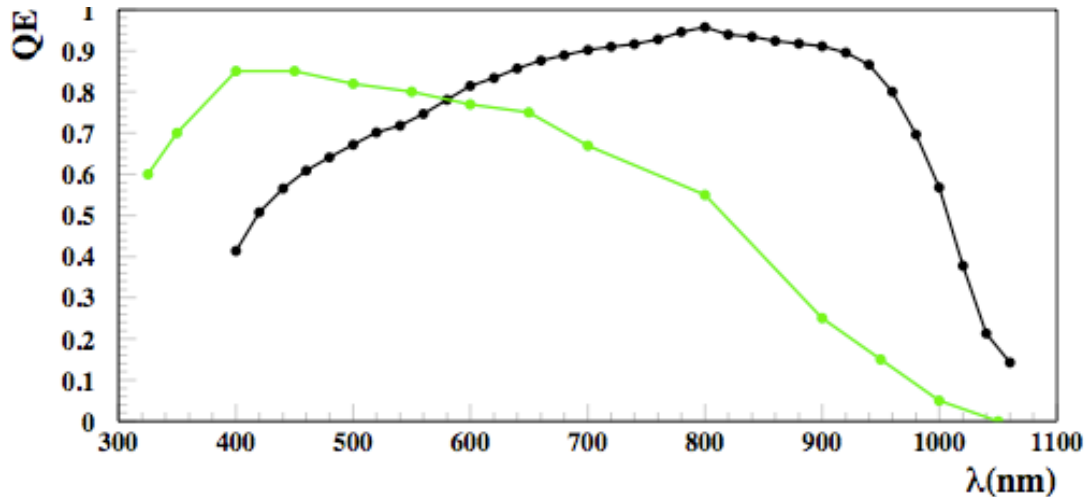

Figure 2: Absolute Quantum Efficiency vs wavelength of the DECam CCDs (black) and the CCDs in Mosaic II (green/grey)

The CCD temperature is $-100 \mathrm{C}$.

The DECam focal plane requires $622 \mathrm{kx} 4 \mathrm{k}$ CCDs for science imaging and $122 \mathrm{kx} 2 \mathrm{k}$ devices for guiding, alignment and focus. The CCDs are fabricated with processing steps at both DASLA SemiConductor and LBNL's Microsystems Lab. Bare silicon die along with cold probe test results are delivered to Fermilab for packaging and testing. Our first prototype packages were produced in 2006 and the design was refined in 2007-8. Production packaging began in 2009. As of May 2010, we have packaged $2092 \mathrm{kx} 4 \mathrm{k}$ CCDs and test results indicate that 88 of them meet the requirements to be called science grade. We have also packaged $232 \mathrm{kx} 2 \mathrm{k}$ CCDs and 13 of them meet the requirements for installation in the focal plane. For more information on the DECam CCDs see Focal plane detectors for the dark energy survey, submitted by Juan Estrada (Paper 7735-61) and Automated Characterization of CCD detectors for DECam, submitted by Donna Kubik (Paper 7735-311) which describe the properties of the DECam CCDs as measured in the CCD packaging and testing facility at Fermilab and the testing procedures that were used to characterize and grade the CCDs.

Figure 3 shows a picture of the DECam focal plane support plate in the imager. The $2 k x 4 k$ science CCDs will be arranged in a hexagon covering an imaging area of 3 sq. degrees. The smaller format CCDs for guiding (G), focusing and alignment (F) are located at the edges of the focal plane and attach to the focal plane support plate with the same footprint and features as the $2 \mathrm{kx} 4 \mathrm{k}$ devices. The active $2 \mathrm{kx} 2 \mathrm{k}$ die covers half of the package. To match the antireflective coating on the CCDs, we use dead CCDs to cover the other half. The $2 \mathrm{kx} 2 \mathrm{k}$ packages are $1.5 \mathrm{~mm}$ thinner than the $2 \mathrm{kx} 4 \mathrm{k}$ packages. For focus and alignment we will position the $2 \mathrm{kx} 2 \mathrm{k}$ devices above and below the focal plane with shims to obtain out of focus donuts. The guide chips will be shimmed to match the height of the science chips. 

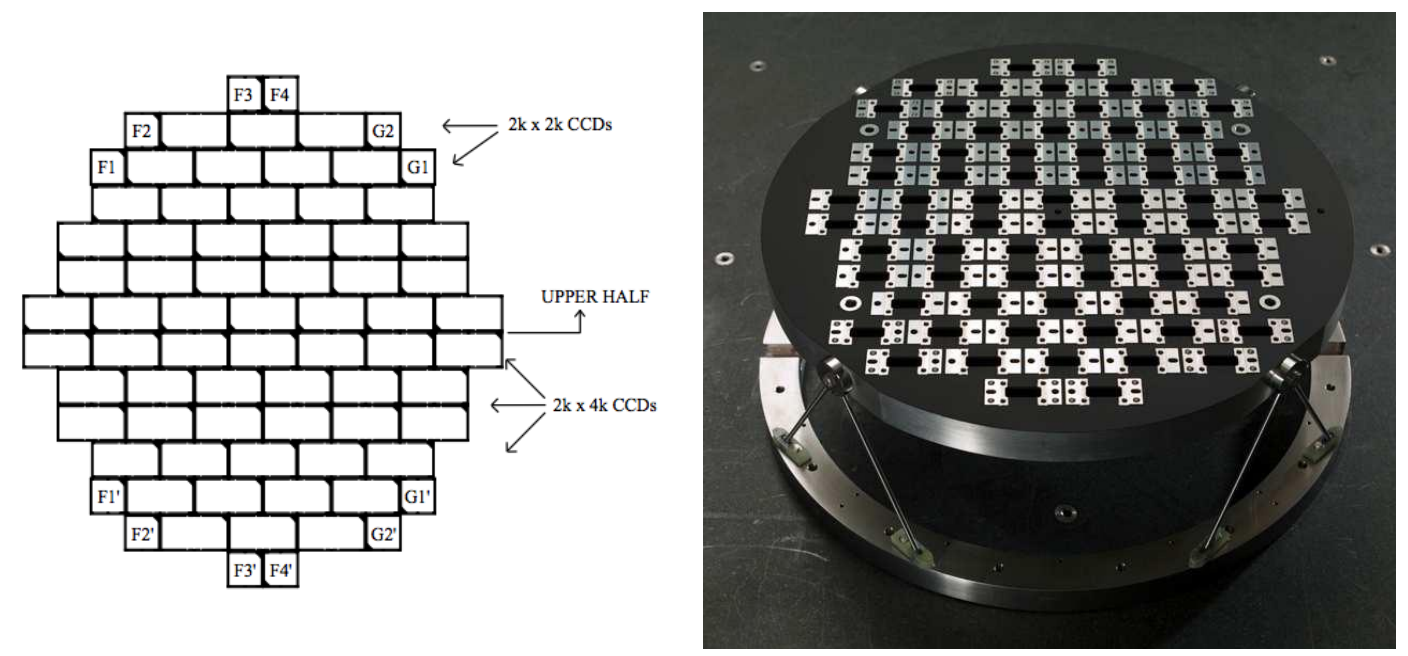

Figure 3: a)The DES Focal plane layout. 2kx2k CCDs labeled as "F" will be used for focus and alignment control; those labeled as "G" will be used for guiding. b) the DECam focal plane support plate The focal plane support plate is painted black to eliminate scattered light between the CCDs. The shiny areas are the contact surfaces for the CCDs.

We plan to read out the alignment and focus CCDs with the image CCDs to provide the possibility for image by image corrections. See Focus and Alignment using Out-of-focus Stellar Images at the Dark Energy Survey submitted by Aaron Roodman (Paper 7735-136), in these proceedings for more details. Currently at the Blanco, observations are interrupted 2-3 times/night to correct the telescope focus and the corrector-primary mirror alignment is checked and adjusted every few months. Experience with DECam on the Blanco will determine the frequency at which corrections are needed to maintain the best image quality.

The DES science requires a uniform PSF. This translates into a requirement that the surface of all the CCDs in the focal plane be within a 60 micron window when the focal plane is a $-100 \mathrm{C}$. Measurement of the focal plane flatness through the window of the vacuum vessel is challenging and we have developed and tested two techniques on the prototype imager. See Measuring the flatness of focal plane for very large mosaic CCD camera submitted by Jiangang Hao (Paper Number: 7735-137) in these proceedings for details.

\subsection{CCD Readout}

CCD testing and characterization is currently being performed at Fermilab using the Monsoon readout system developed originally by NOAO (see www.noao.edu/etc/monsoon for additional information on the Monsoon system). We have modified/customized all of electronics boards and crates to meet specific DES requirements. In particular, as a prime focus instrument with tight space and thermal constraints we have developed a compact system that can readout 72 CCDs using three thermally controlled crates and 6 independently controlled backplanes. A water-glycol mixture, will be transported to these crates through insulated lines to keep the boards at a constant temperature and to remove the heat from the electronics. See System architecture of the dark energy survey camera readout electronics submitted by Terri Shaw (Paper 7735-123) and Readout Electronics for DECam submitted by Javier Castilla (Paper 7735-311) in these proceedings. Fabrication of all of the DECam boards is nearly complete and testing is in progress.

A by-product of our CCD acquisition strategy is a significant number of engineering and mechanical grade devices that are ideal for testing and integration. With our full size prototype imager we have performed many studies of multiple CCD readout. With 26 engineering grade CCDs installed, and CCD readout distributed over 2 separate crates, we have demonstrated routine operation and acquisition of images at 250 kpixels per second with less than $10 \mathrm{e}$ noise, thus beating the DECam requirements. 


\subsection{Shutter}

The DECam shutter was designed and built by the Argelanger Institute for Astronomy and Bonn University. The shutter has sliding blades (a "flying slit") for fast exposures and to obtain equal exposure times in both directions of travel. It is the largest shutter they have built and it exceeds all of the DECam requirements. Figure 4 shows the shutter and Table 2 shows the a sample of the requirements and performance achieved.
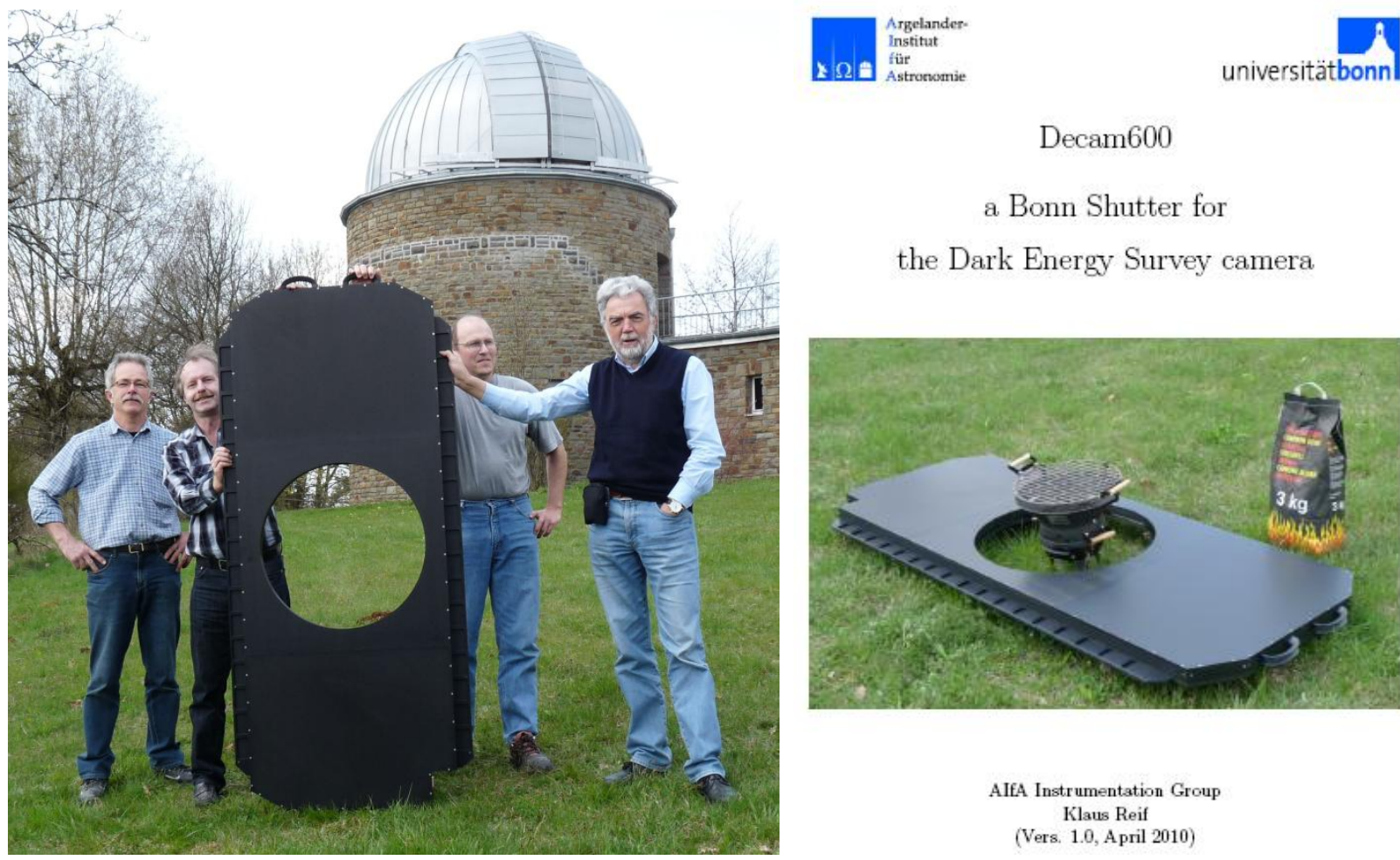

\section{Decam600}

a Bonn Shutter for

the Dark Energy Survey camera

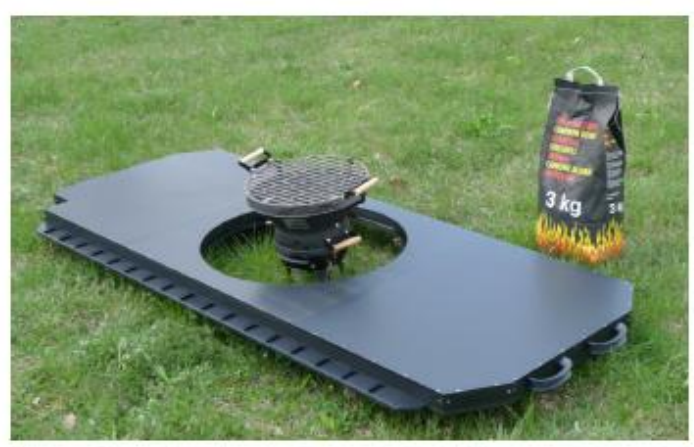

$$
\begin{gathered}
\text { AIfA Instrumentation Group } \\
\text { Klaus Reif } \\
\text { (Vers. 1.0, A pril 2010) }
\end{gathered}
$$

Figure 4: the DECam shutter at the Hoher List Observatory

Table 2 DECam requirements for the shutter and the results obtained with the Bonn shutter.

\begin{tabular}{|l|c|c|}
\hline Item & Requirement & Test result \\
\hline Aperture diameter & $600 \mathrm{~mm}$ & $600 \mathrm{~mm}$ \\
\hline Mass & $<36 \mathrm{~kg}$ & $31.5 \mathrm{~kg}$ \\
\hline \hline Exposure time uniformity & better than $10 \mathrm{msec}$ & better than $1 \mathrm{msec}$ \\
\hline Exposure time repeatability & better than $5 \mathrm{msec}$ & $6 \mu \mathrm{sec}$ (stddev) \\
\hline Exposure time accuracy & better than $50 \mathrm{msec}$ & $6 \mu \mathrm{sec}(\mathrm{stddev})$ \\
\hline Shutter dead time & $\leq 1 \mathrm{sec}$ & $1.049 \mathrm{sec}$ \\
\hline Absolute timing & measurable to $<10 \mathrm{msec}$ & $<1 \mathrm{msec}$ \\
\hline Min. exposure time & $10 \mathrm{msec}$ & $\approx 300 \mu \mathrm{sec}$ \\
\hline \hline Light tightness & $-5000 \mathrm{phot} / \mathrm{h} / \mathrm{pix} @ 300 \mathrm{lux}$ & $25 \mathrm{phot} / \mathrm{h} / \mathrm{pix}$ \\
\hline \hline Performance range & $-5 \mathrm{C}$ to $25 \mathrm{C}$ & $-5 \mathrm{C}$ to $25 \mathrm{C}$ \\
\hline Heat dissipation & $300 \mathrm{~mW}+15 \mathrm{Ws} \mathrm{per} \mathrm{expo.}$ & o.k. \\
\hline MTBF & $1,250,000$ cycle test with model & passed \\
\hline \hline Exposure uniformity & (see Ref. 4.1$)$ & better than $1 \mathrm{msec}$ \\
\hline Blade position consistency & shall be reported & 2 steps or better \\
\hline Back and forth symmetry test & (see Ref. 4.1 ) & better than $1 \mathrm{msec}$ \\
\hline Positioning accuracy & known to an accuracy of $10 \mathrm{msec}$ & about $1 \mathrm{msec}$ \\
\hline
\end{tabular}




\subsection{Optics}

The final optical corrector design was produced by Rebecca A. Bernstein, UC Observatories \& Astrophysics Dept, UC Santa Cruz, CA. It consists of five fused silica lenses that create an unvignetted $2.2^{\circ}$ diameter image area. The largest element (C1) is $930 \mathrm{~mm}$ diameter. The last element (C5) is 540mm in diameter and serves as the window on the CCD vacuum vessel. A schematic of the corrector elements is shown in Figure 5. The large space between elements 3 and 4 allows room for the Bonn shutter and 4 filter cassettes. Each cassette can hold two filters and can slide the filter in and out of the optical path. The shutter is located between $\mathrm{C} 4$ and the filter cassettes.

The optical design and fabrication plan for the DES corrector is described in reference [2]. As-built features of the lenses are being incorporated into the optical design by Sue Worswick under contract to University College London. These small deviations from the specifications can be accommodated by adjustments in the spacing between the lenses without reducing the image quality.

The lenses will be mounted into cells and then into the corrector barrel. Figure 6 shows the $\mathrm{C} 4$ cell under inspection at University College London and the C3 lens ready for inspection at SESO in April. Both passed inspection and meet the DECam requirements. Fabrication of the $\mathrm{C} 1, \mathrm{C} 2$ and $\mathrm{C} 3$ cells is nearly complete with an expected delivery to UCL in June 2010.

We have developed a plan for alignment of the cells and barrel that minimizes the handling of the lenses: after inspection at UCL, the C1-C4 cells will be shipped to Fermilab for alignment in the corrector barrel. The cells will be pinned to the barrel and then the barrel-cell assembly shipped back to UCL. UCL will remove the cells from the barrel and install the lens into the cells. UCL will install the lens-cell assemblies into the corrector barrel and check the optical alignment. If necessary it is possible to reposition the lens-cell assemblies in the barrel, however, the goal of the alignment of the cells at Fermilab is to eliminate or minimize the need for adjustment after the lenses are in the cells. The C5 cell is complete and undergoing fit tests to the imager and corrector barrel at Fermilab. It will be shipped along with the barrel and the rest of the cells to UCL for integration with the C5 lens.

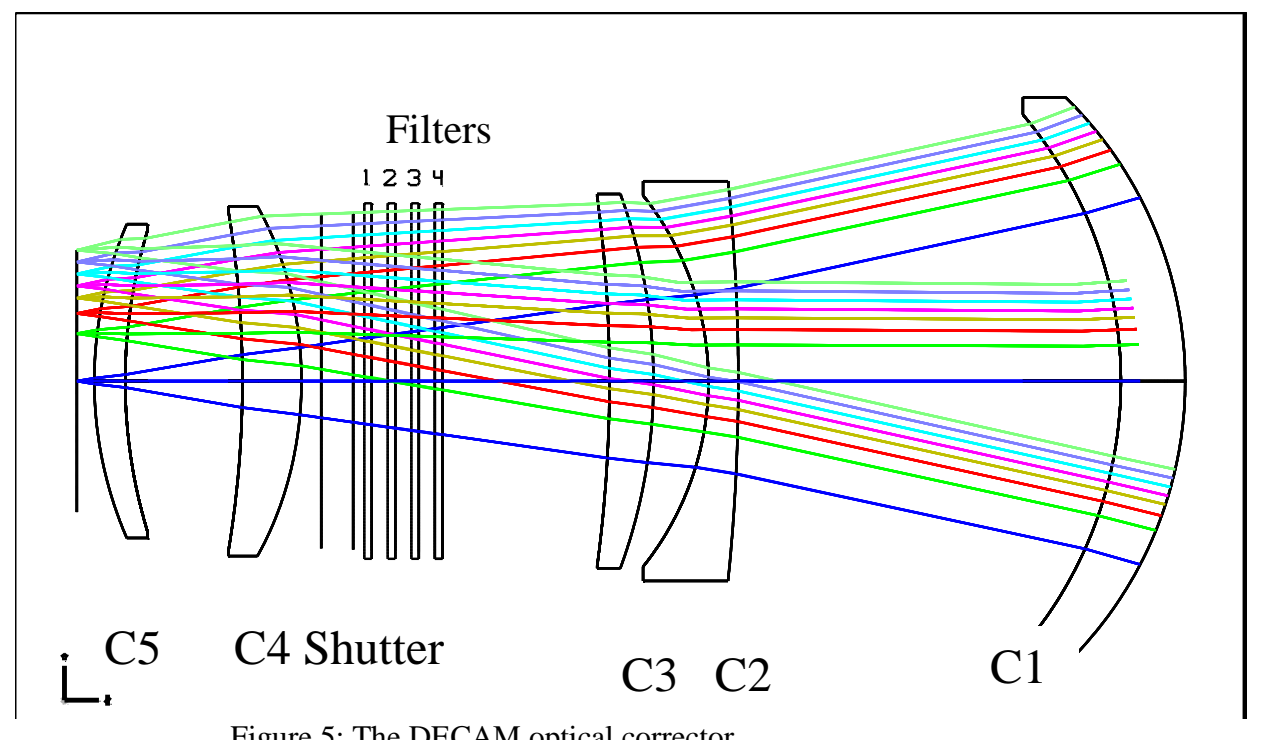

Figure 5: The DECAM optical corrector 


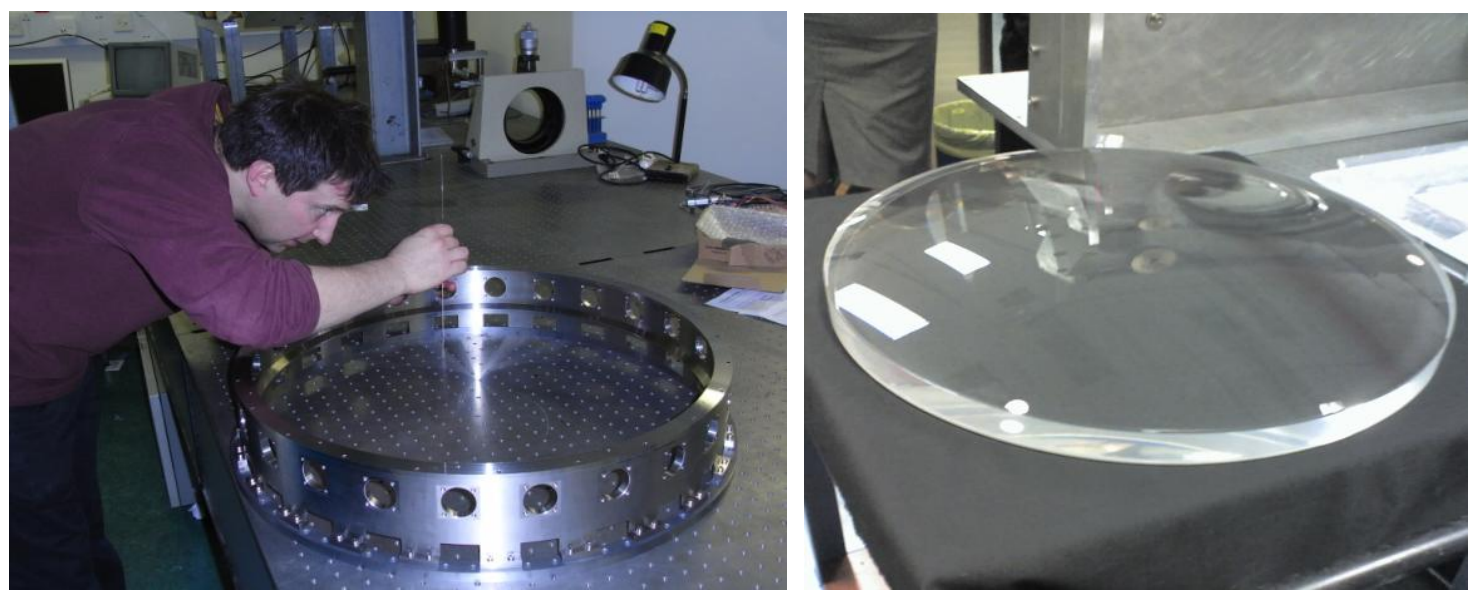

Figure 6 The C4 cell at University College London and the C3 lens ready for inspection at SESO.

\subsection{Corrector Barrel}

A solid model of the corrector barrel is shown in Figure 6. The corrector barrel consists of two large pieces that bolt together and a protective shroud for $\mathrm{C} 1$. A conical section supports $\mathrm{C} 1$ and is keyed and bolted to the body section. The body section provides the interface to the hexapod and supports C2- C5, the imager, the filter cassettes and the shutter. The corrector barrel is made of steel for ease of machining and welding. Tight flatness and parallelism tolerances are imposed on the surfaces for mounting the lens cells. Two barrels have been ordered; the one with the best match to our requirements will be used as the DECam corrector. The other barrel will be used for tests on the telescope simulator at Fermilab. Figure 7 shows two sections of the first barrel awaiting inspection on the coordinate measuring machines at Fermilab. Delivery of the second barrel is expected in June 2010.

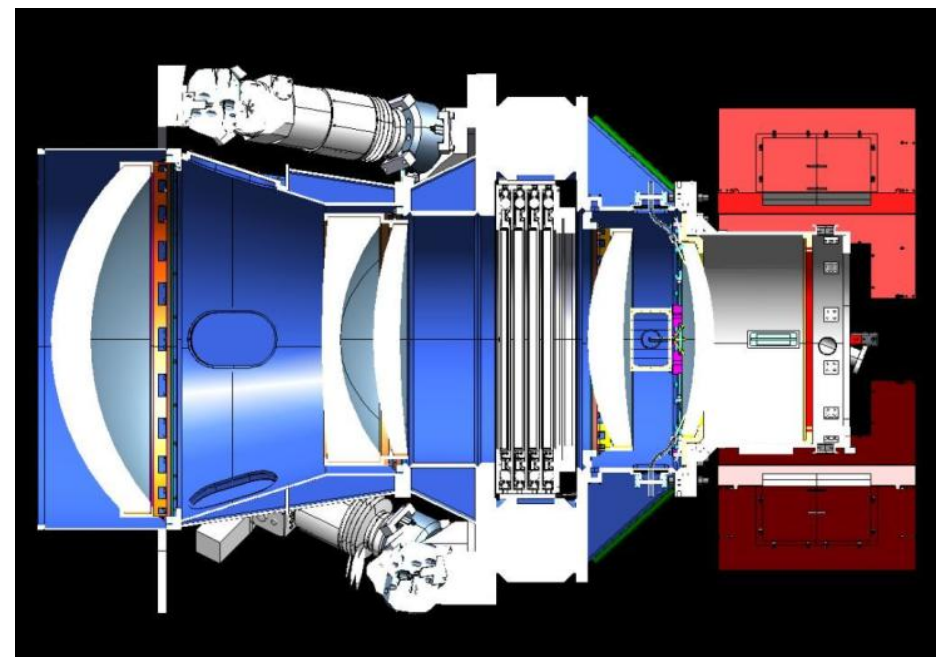

Figure 6: Conceptual Design of the DES Corrector Barrel 

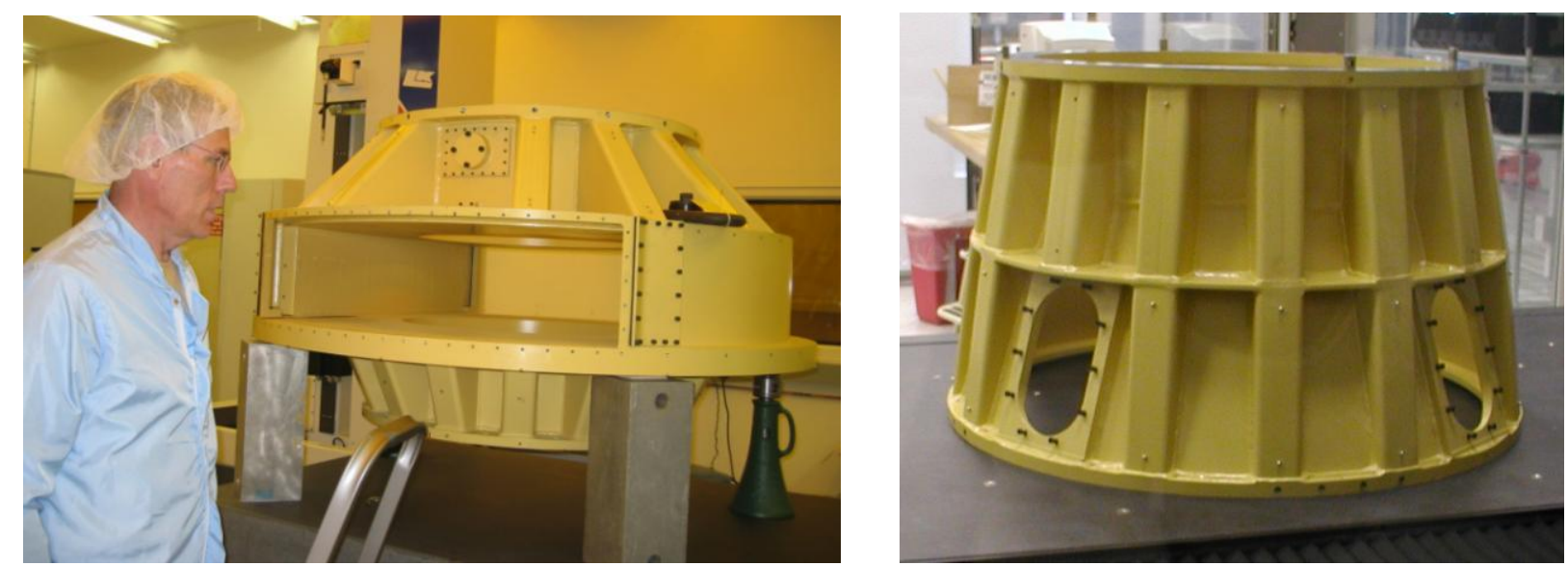

Figure 7 Left the body of the corrector which houses lenses C4-C2, the filter changer and the shutter. Right: the conical piece of the corrector which attaches to the body and supports C1. Inspection of the delivered parts is currently in progress at Fermilab.

\subsection{Filter Changer}

The filter exchange mechanism can hold 8 filters in four cassettes ( 2 filters/ cassette) which are oriented perpendicular to the optical axis. Figure 8 shows one cassette with mass models installed for testing and the 4 cassette assembly ready for shipping to Fermilab. The cassettes use pneumatics to position each filter in or out of the optical path. Each cassette can accept a filter substrate that is $620 \mathrm{~mm}$ diameter and $13-14 \mathrm{~mm}$ thick. The beam is nearly collimated in the areal of the filters and thus all the DES filters will be the same size with a clear aperature of $600 \mathrm{~mm}$. See Large format filter changer mechanism and shutter for the dark energy survey submitted by Greg Tarle (Paper 7739-162), for more details. The filter changer and shutter are installed in the corrector barrel after the cage-barrel assembly is mounted at the prime focus of the telescope. Individual filters are installed in or removed from the cassettes using a custom box that can bolt to either end of the cassette. We will have the five DES filters for the beginning of the survey and NOAO may later provide additional filters for community use.

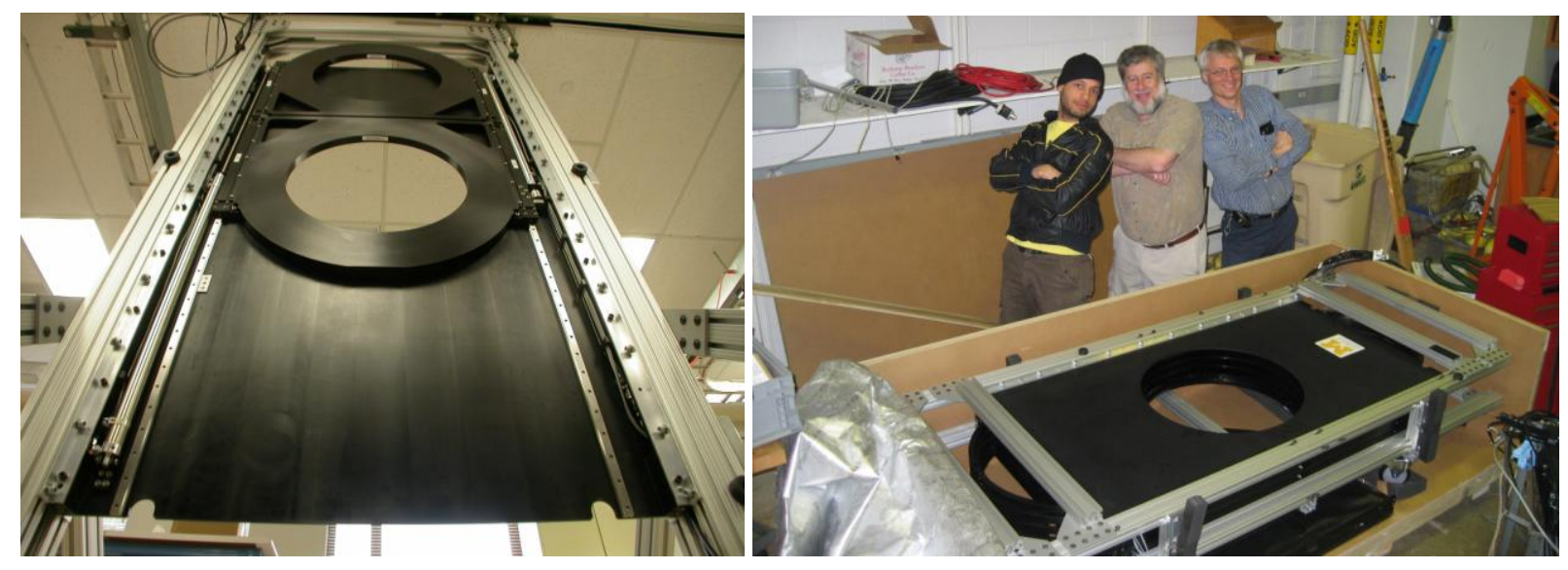

Figure 8 a) One filter changer cassette with mass models installed for testing b) Assembled 4 cassette filter changer ready for shipping to Fermilab.

\subsection{Filters}

The DECam filters push the limits of filter manufacturers due to our large clear aperture $(600 \mathrm{~mm})$ and tight uniformity requirements (see table 3). Asahi Spectra Co., of Tokyo, Japan is under contract with Fermilab for fabrication of the 5 DECam filters. Asahi has assembled a large coating chamber ( $\sim 3 \mathrm{~m}$ diameter, see Figure 9$)$ and are currently achieving 
good uniformity on witness samples. Coating of the DECam filters will begin in July 2010 and completion of all the filters is expected before the end of calendar 2010.

Table 3: Approximate characteristics of $620 \mathrm{~mm}$ diameter DES Filters

\begin{tabular}{|c|c|c|c|c|}
\hline Filter & $\begin{array}{c}\text { Center wavelength } \\
(\mathrm{nm})\end{array}$ & $\begin{array}{c}\text { FWHM } \\
(\mathrm{nm})\end{array}$ & $\begin{array}{c}\text { Average absolute } \\
\text { transmission }\end{array}$ & $\begin{array}{c}\text { Allowable gradient in } \\
\text { transmission curve }\end{array}$ \\
\hline DES g & 475 & 150 & $>85 \%$ & $\pm 5 \%$ \\
\hline DES r & 635 & 150 & $>85 \%$ & $\pm 7 \%$ \\
\hline DES i & 775 & 150 & $>85 \%$ & $\pm 5 \%$ \\
\hline DES z & 925 & $>100$ & $>85 \%$ & $\pm 9 \%$ \\
\hline DES y & Approx. 1000 & $>85 \%$ & $\pm 9 \%$ \\
\hline
\end{tabular}
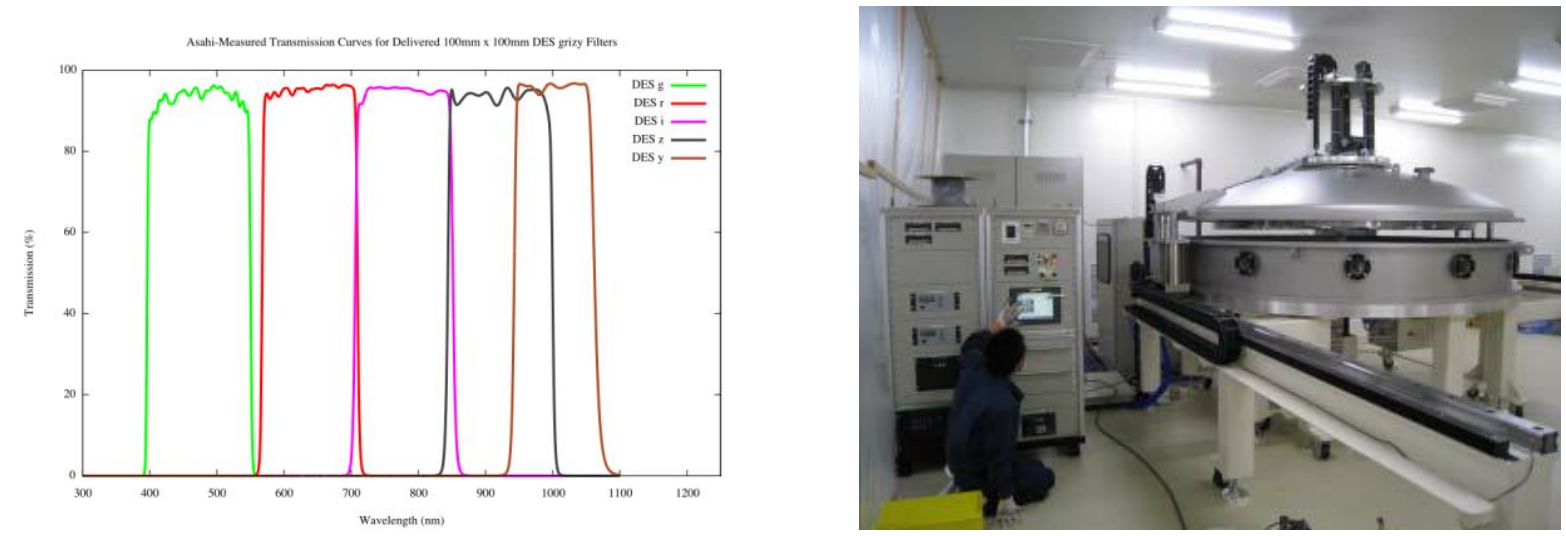

Figure 9 a) DES filter bandpasses achieved by Asahi on 100mm square filters b)Asahi engineer raises the cover of the $\sim 3 \mathrm{~m}$ diameter magnetron sputtering coating chamber that will be used for the DECam filters.

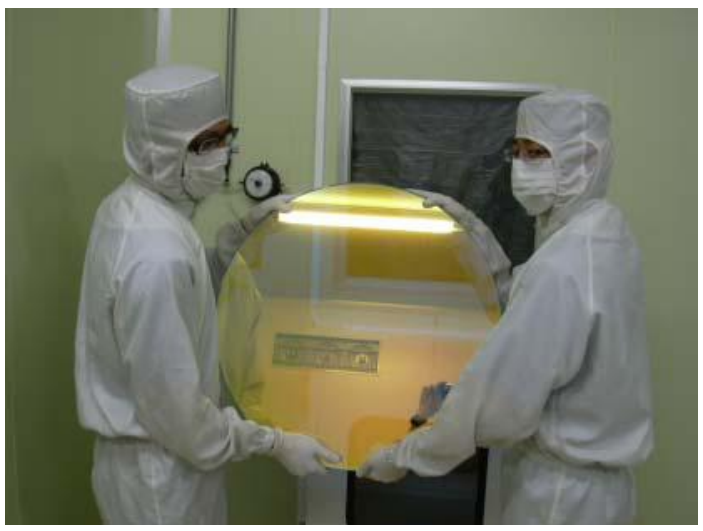

Figure 10 Asahi personnel hold a 620mm diameter glass substrate which has been coated with a short pass film (24 layers).

\subsection{Alignment and Focus}

The support and position adjustment of the camera/barrel assembly in the prime focus cage incorporates a hexapod capable with accurate 6-axis motion. The base of the hexapod will be mounted to a ring built into the prime focus cage. The corrector will be attached to the mobile flange of the hexapod using the front plate of the corrector body. Note that the CCD vessel is aligned and bolted to the corrector and the combination camera/barrel system moves as a single unit. 
The hexapod system will provide both the focus adjustments and the capability to keep the corrector aligned to the primary mirror. The large load $(3500 \mathrm{~kg})$ and tight positioning requirements presented a challenge to traditional hexapod designs. ADS International, of Valmadrera, Italy, has developed and tested a new hexapod for DES. The design uses large, modular flex joints to attach the hexapod actuators to the mobile and fixed flanges. Figure 11 shows the complete hexapod at the ADS facility. Table 4 show the DECam requirements and the results achieved at ADS with the assembled hexapod. Delivery to Fermilab is expected in June 2010.

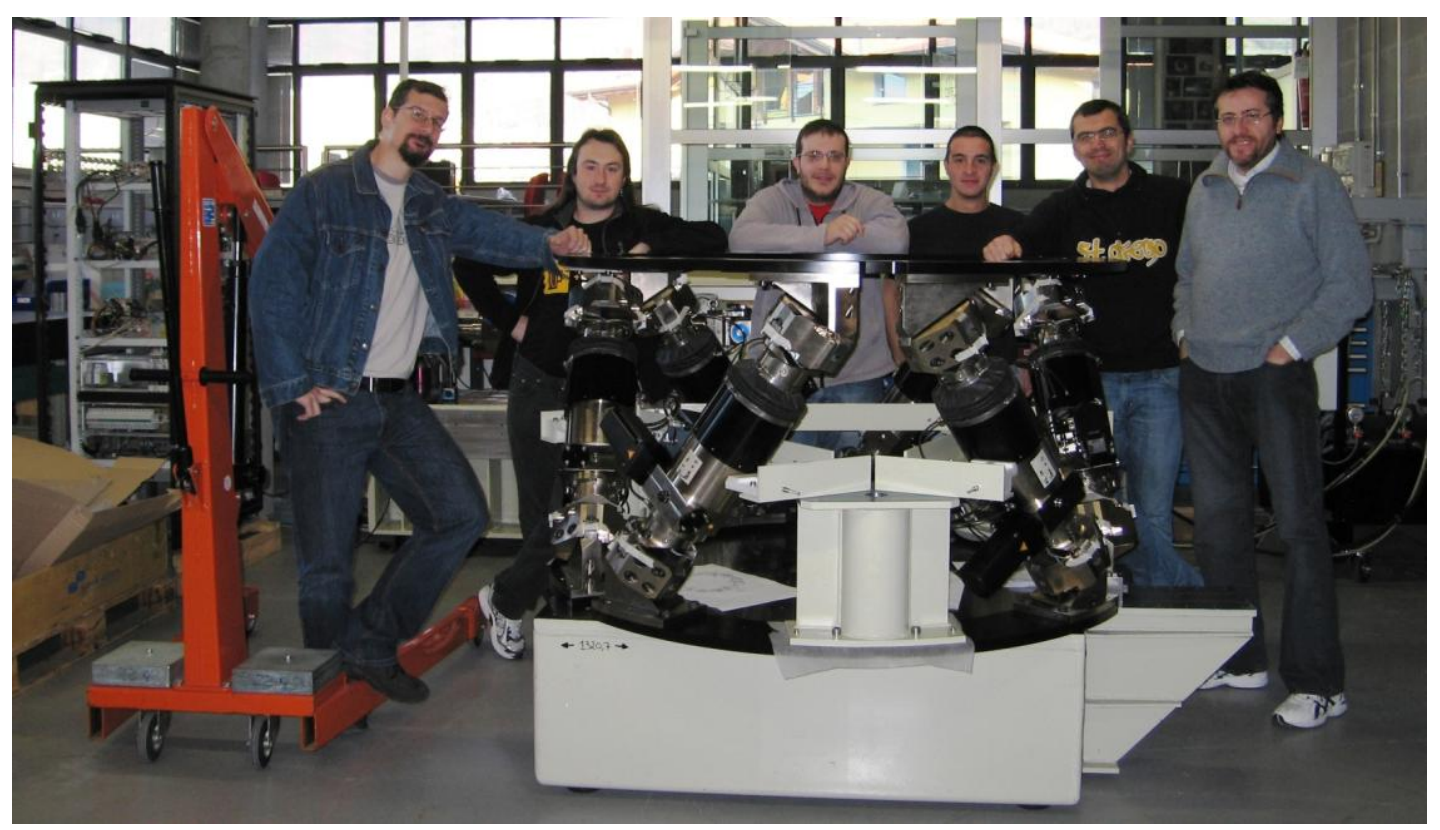

Figure 11 the assembled hexapod at ADS International, Valmadrera, Italy. The fixed flange is on the bottom. The assembly fixture is also shown.

The focus and alignment CCDs will be read out with the image CCDs providing image-by-image information on the telescope focus and the alignment of the corrector to the primary mirror. Comparison of the out-of-focus images above and below the focal plane will indicate the direction of motion needed to bring the image back into focus. Out of focus image data has been collected with the Mosaic camera on the Blanco for development of the DES donut analysis. See Focus and Alignment using Out-of-focus Stellar Images at the Dark Energy Survey submitted by Aaron Roodman (Paper 7735-136), for more details. We also plan to install a laser-alignment system, similar to BCAM systems (see alignment.hep.brandeis.edu/Devices/BCAM/), which will directly measure the relative positions of the mirror and the corrector without needing to operate the CCDs. Information from one or both of these systems will be used to determine if the corrector needs to be moved laterally in the cage to be better aligned with the primary mirror.

Table 4 Focus range and resolution for the DECam hexapod.

\subsection{Focus range}

\begin{tabular}{|c|c|c|c|}
\hline Item & Spec. Value & Compliance & Comment \\
\hline \multicolumn{4}{|c|}{$Z$ (focus) } \\
\hline 4.4.2.3 - Focus range & $3 \mathrm{~mm}$ & C & \\
\hline $\begin{array}{l}4.4 .2 .4-\text { Peak-Peak error on focus } \\
\text { range }(3 \mathrm{~mm})\end{array}$ & $\pm 7.5 \mu \mathrm{m}$ & C & $\begin{array}{l}\text { Error }<1.8 \text { um ptv at } \\
\text { constant load }\end{array}$ \\
\hline $\begin{array}{l}4.4 .2 .5 \text { - Lateral drift limit on focus } \\
\text { range }(3 \mathrm{~mm})\end{array}$ & $\pm 25 \mu \mathrm{m}$ & c & $\begin{array}{l}\text { drift in } X<3.8 \text { um ptv } \\
\text { drift in } Y<3.5 \text { um ptv }\end{array}$ \\
\hline $\begin{array}{l}\text { 4.4.2.6 - Tilt drift limit on focus range } \\
(3 \mathrm{~mm})\end{array}$ & \pm 3 arcsec & C & $\begin{array}{l}\text { Drift in TIP }<0.3 \text { um ptv } \\
\text { Drift in TILT }<0.5 \text { um ptv }\end{array}$ \\
\hline $\begin{array}{l}\text { 4.4.2.7 - Twist drift limit on focus range } \\
(3 \mathrm{~mm})\end{array}$ & \pm 3 arcsec & C & By design \\
\hline $\begin{array}{l}4.4 .2 .8 \text { - Time to make } 0.15 \mathrm{~mm} \\
\text { position change }\end{array}$ & $7.5 \mathrm{sec}$ & C & $\begin{array}{l}\text { Time to make } 0.1 \mathrm{~mm} \text { is } \\
0.6 \mathrm{sec} \text {. } \\
\text { Estimated time to make } \\
0.15 \mathrm{~mm} \text { is }<1 \mathrm{sec}\end{array}$ \\
\hline
\end{tabular}




4.2 Focus resolution
\begin{tabular}{|l|c|c|l|}
\hline Item & Spec. Value & Compliance & Comment \\
\hline \multicolumn{4}{|c|}{ (focus) } \\
\hline $\begin{array}{l}4.4 .2 .9 \text { - resolution (min incremental } \\
\text { motion) }\end{array}$ & $\begin{array}{c}1 \mu \mathrm{m}( \pm 10 \% \\
\text { tolerance) }\end{array}$ & $\mathrm{C}$ & $\begin{array}{l}\text { Results } 1 \mathrm{um}( \pm 5 \% \\
\text { tolerance) }\end{array}$ \\
\hline
\end{tabular}

\subsection{The cage}

The DECam cage provides the attachment point to the telescope fins and the base support for the hexapod fixed flange. Each rail has 3 holes for the pins that attach the fins to the cage. To electrically isolate the DECam cage from the telescope, we will install 0.030 " thick G10/FR4 isolation bushings in the holes in the fins. The cage also provides the interface for the F/8 mirror cell.

Figure 12 shows the completed cage at Fermilab (black), along with the lifting fixture (yellow). Swivel hoists can attach to the lifting fixture at the center of mass of the cage. This allows rotation of the cage from horizontal to vertical which is required for installation on the telescope and the telescope simulator.
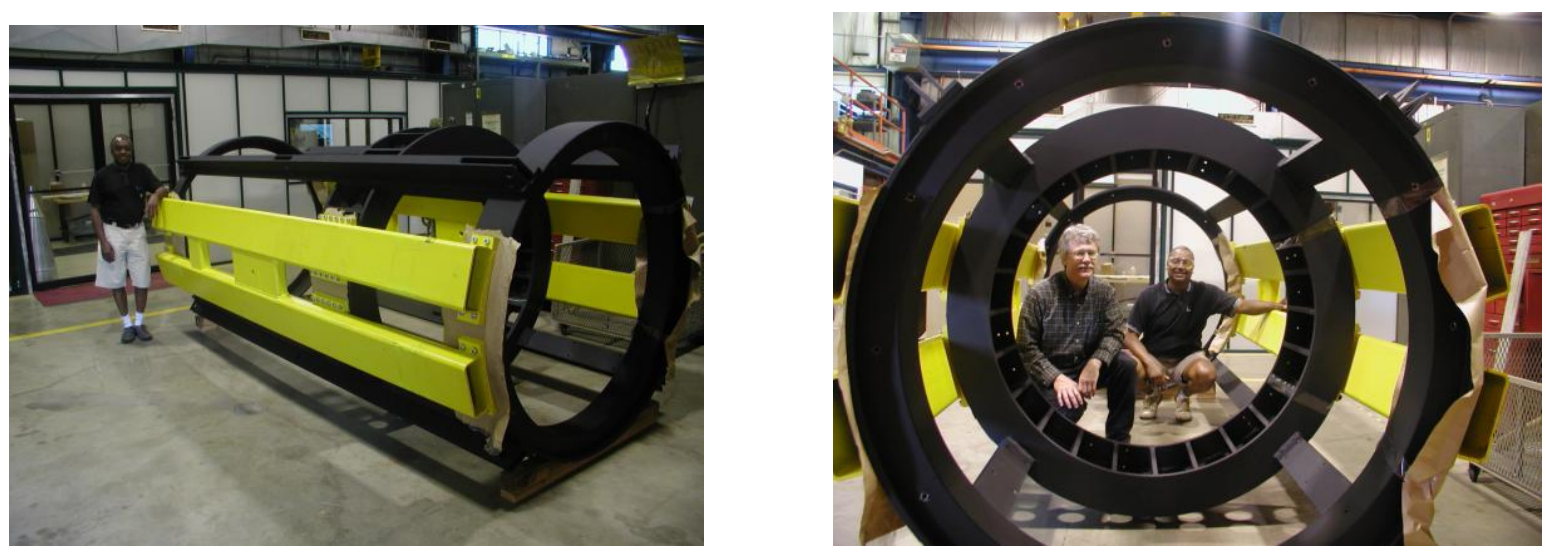

Figure 12 The DECam Cage(black) and lifting figure (yellow)

\subsection{Survey Image System Process Integration (SISPI)}

The mountain top software in DES is called Survey Image System Process Integration (SISPI). This is the set of processes that will control the image acquisition and deliver the images to the data management system for processing. DES will have over a dozen computers on the mountain devoted to different aspects of the SISPI. Prototype versions of the SISPI code are in constant use with the prototype imager, providing valuable feedback and debugging opportunities. See The DECam data acquisition and control system submitted by Klaus Honscheid (Paper 7740-57) and HTML 5, Websockets, and Sproutcore: a web-based user interface for the dark energy camera (DECam) submitted by Jacob Eiting (Paper 7740-39) in these proceedings for more details.

\section{DECAM SCHEDULE}

All of the major components of DECam are complete or nearly complete, including a telescope simulator which matches the top end of the Blanco telescope. Over the next few months we will be assembling DECam, without the optics, and installing it on the telescope simulator. The cage, hexapod, barrel, filter changer and shutter will be installed and operated prior to installation of the CCD imager. By August 2010, the CCD imager with 30 engineering grade CCDs, will be install on the telescope simulator using the same fixtures we will use at CTIO. Full systems tests in different orientations will take $\sim 4$ months to complete. By Jan. 2011 we expect to remove the imager from the telescope simulator and begin installation of the science grade devices. In parallel, those systems not needed for the science grade CCD installation and testing will be shipped to CTIO in Jan. 2011 for installation and commissioning. By June 2011 we expect the CCD imager to arrive at CTIO and be ready for installation on the Blanco by the end of August. First light on the Blanco and the beginning of commissioning are expected in Oct. 2011. 


\section{CONCLUSIONS}

The Dark Energy Survey Collaboration is building a new powerful CCD camera to measure the dark energy equation of state parameter, w, using 4 complementary techniques. The 3 sq. deg camera will be 7 times larger and 7 times faster than the prime focus camera currently on the Blanco and will use CCDs that are $\sim 10$ times more sensitive in the z-band that traditional CCDs. The DECam design has focused on maximizing science potential while minimizing development time and costs. To minimize the downtime of the Blanco for DECam installation and commissioning, a significant portion of the project is devoted to integration and testing prior to shipping to CTIO. With most of the major systems nearly complete, the integration and testing phase of the project is now in progress now at Fermilab. The Dark Energy Survey is expecting to begin commissioning with DECam on the Blanco in October 2011.

\section{AKNOWLEDGEMENTS}

Funding for the DES Projects has been provided by the U.S. Department of Energy, the U.S. National Science Foundation, the Ministry of Science and Education of Spain, the Science and Technology Facilities Council of the United Kingdom, the Higher Education Funding Council for England, the National Center for Supercomputing Applications at the University of Illinois at Urbana-Champaign, the Kavli Institute of Cosmological Physics at the University of Chicago, Financiadora de Estudos e Projetos, Fundação Carlos Chagas Filho de Amparo à Pesquisa do Estado do Rio de Janeiro, Conselho Nacional de Desenvolvimento Científico e Tecnológico and the Ministério da Ciência e Tecnologia, the German Research Foundation-sponsored cluster of excellence "Origin and Structure of the Universe" and the Collaborating Institutions in the Dark Energy Survey.

The Collaborating Institutions are Argonne National Laboratories, the University of California at Santa Cruz, the University of Cambridge, Centro de Investigaciones Energeticas, Medioambientales y Tecnologicas-Madrid, the University of Chicago, University College London, DES-Brazil, Fermilab, the University of Edinburgh, the University of Illinois at Urbana-Champaign, the Institut de Ciencies de l'Espai (IEEC/CSIC), the Institut de Fisica d'Altes Energies, the Lawrence Berkeley National Laboratory, the Ludwig-Maximilians Universität, the University of Michigan, the National Optical Astronomy Observatory, the University of Nottingham, the Ohio State University, the University of Pennsylvania, the University of Portsmouth, SLAC, Stanford University, and the University of Sussex.

\section{REFERENCES}

[1] P. Doel et al, "Design and status of the optical corrector for the DES survey instrument" Proc. SPIE, Vol. 7014, 70141V (2008)

[2] Holland, S. et al. "Fully Depleted, Back-Illuminated Charge-Coupled Devices Fabricated on High-Resisitivity Silicon," IEEE Trans. Elec. Dev., 50, 225 (2003) 